\title{
Failure of Anisotropic Unstructured Mesh Adaption Based on Multidimensional Residual Minimization
}

\author{
William A. Wood* and William L. Kleb* \\ NASA Langley Research Center, Hampton, VA 23681
}

\begin{abstract}
An automated anisotropic unstructured mesh adaptation strategy is proposed, implemented, and assessed for the discretization of viscous flows. The adaption criteria is based upon the minimization of the residual fluctuations of a multidimensional upwind viscous flow solver. For scalar advection, this adaption strategy has been shown to use fewer grid points than gradient based adaption, naturally aligning mesh edges with discontinuities and characteristic lines [Wood, AIAA 99-3254]. The adaption utilizes a compact stencil and is local in scope, with four fundamental operations: point insertion, point deletion, edge swapping, and nodal displacement. Evaluation of the solution-adaptive strategy is performed for a two-dimensional blunt body laminar wind tunnel case at Mach 10. The results demonstrate that the strategy suffers from a lack of robustness, particularly with regard to alignment of the bow shock in the vicinity of the stagnation streamline. In general, constraining the adaption to such a degree as to maintain robustness results in negligible improvement to the solution. Because the present method fails to consistently or significantly improve the flow solution, it is rejected in favor of simple uniform mesh refinement.
\end{abstract}

\section{Nomenclature}

\begin{tabular}{ll}
$\vec{A}, \overrightarrow{\mathcal{A}}$ & Flux Jacobian in conserved or auxiliary variable \\
$a$ & Speed of sound \\
$F$ & Flux of conserved quantities \\
$I$ & Identity matrix \\
$\hat{\imath}, \hat{\jmath}$ & Cartesian unit vectors \\
$\ell$ & Edge length \\
$\mathbf{M}$ & Upwinding matrix \\
$\hat{n}$ & Outward-normal unit vector \\
$P$ & Pressure \\
$S$ & Area \\
$s$ & Entropy: $d s=d \rho-d P / a^{2}$ \\
$T$ & Temperature \\
$U$ & Conserved variables \\
$u, v$ & Velocity components \\
$\vec{V}$ & Velocity vector \\
$\mathrm{W}$ & Auxiliary variables: $d W=(d s, \tilde{\rho} d \vec{V}, d P)$ \\
$x, y$ & Cartesian coordinates \\
$\mathrm{Z}$ & Parameter vector: $\sqrt{\rho}(1, u, v, H)$ \\
$\alpha, \beta$ & Generalized wave speeds in $\xi, \eta$ directions \\
$\gamma$ & Ratio of specific heats \\
$\xi$ & Curvilinear coordinate on triangle edge $\overline{12}$ \\
$\eta$ & Curvilinear coordinate on triangle edge $\overline{23}$ \\
$\Upsilon$ & Minimization functional \\
$\phi, \breve{\phi}$ & Fluctuation in conserved or auxiliary variables \\
$\rho$ & Density \\
$\Xi$ & Functional weighting matrix \\
$\Omega$ & Integration element \\
\hline
\end{tabular}

*Aerospace Engineer, Aerothermodynamics Branch, Aerodynamics, Aerothermodynamics, \& Acoustics Competency. AIAA member.

This material is a work of the U.S. Government and is not subject to copyright protection in the United States.
Superscripts:

()$^{T} \quad$ Transpose

()$^{v} \quad$ Viscous component

()$^{x, y}$ Spatial component of a vector

Subscripts:

()$_{i} \quad$ Node number

( )T Triangle

Over-bars indicate linear averages and tildes indicate average values assuming linear variation of the parameter vector.

Variables lacking explicit dimensions are in nondimensional form, normalized by the free stream values of density, velocity, viscosity, and temperature along with a characteristic length, as appropriate.

\section{Introduction}

$\mathbf{T}$ HOMAS et al. ${ }^{1}$ identify viscous flow unstructured mesh adaptation as a critical enabling technology for the inclusion of high fidelity computational fluid dynamics in the vehicle design cycle. The development of robust automated unstructured grid generation and adaption methods will directly reduce the time required for both the initial grid generation for complex vehicles and the solution computation for complex flow fields. Notable achievements in this field of unstructured mesh adaption have recently been demonstrated by Venditti and Darmofal, ${ }^{2}$ using adjoints to optimize the mesh distribution, and by Habashi et al., ${ }^{3,4}$ using anisotropic feature adaption.

Concurrent work in flow solvers for hypersonic aerothermodynamic applications has shown promise 
for nonlinear multidimensional upwind fluctuation splitting schemes as being more accurate and less grid sensitive than traditional locally one-dimensional flux splitting schemes. ${ }^{5-7}$ Unstructured mesh adaption based upon the minimization of the fluctuation splitting distribution has shown some remarkable natural alignment properties for linear ${ }^{8,9}$ and nonlinear ${ }^{10}$ model problems, but has not been demonstrated for the full system of equations for viscous fluid dynamics. Alignment of the mesh to the bow shock has been shown to be a critical factor in the accuracy of hypersonic solutions, ${ }^{11}$ and the successful extension of the natural alignment tendency inherent in fluctuation minimization to a high speed fluid dynamics capability could be the desired breakthrough to the cost barrier of including computational fluid dynamics in the vehicle design cycle.

This paper presents an automated anisotropic unstructured mesh adaption process based upon the minimization of distributions for a nonlinear multidimensional upwind fluctuation splitting scheme. The adaption is local in scope and thus convenient to parallelize. The demonstration case is for perfect gas two-dimensional Mach-10 flow over a sting-mounted entry capsule profile.

\section{Flow Solver}

The flow solver is a nonlinear monotone secondorder accurate multidimensional upwind fluctuation splitting scheme as introduced by Sidilkover ${ }^{12}$ with extensions to viscous flows. ${ }^{13}$ The solver produces exact convective solutions along characteristics on unstructured grids where one edge of each cell is aligned with the cell-averaged transport direction. ${ }^{10}$ This characteristic alignment property of the flow solver is the basis for driving the mesh adaption strategy described in the following section. Traditional locally one-dimensional flux splitting solvers do not share this method-ofcharacteristics property.

While a full description of the second-order accurate viscous flow solver is available in Ref. 6, including verification and validation results, the first-order discretization is briefly sketched here to provide context for the residual derivatives that follow in the adaptation section. The equations of motion are written in vector form as

$$
U_{t}=-\vec{\nabla} \cdot \vec{F}+\vec{\nabla} \cdot \vec{F}^{v}
$$

where $\vec{F}$ and $\vec{F}^{v}$ are the inviscid and viscous fluxes and $U$ is the vector of conserved variables. Integrating over a triangular element, Figure 1, the right-hand side (RHS) can be expressed in terms of inviscid and viscous fluctuations, $\phi$ and $\phi^{v}$, as

$$
\mathrm{RHS}=-\int_{\Omega} \vec{\nabla} \cdot \vec{F} d \Omega+\int_{\Omega} \vec{\nabla} \cdot \vec{F}^{v} d \Omega=\phi+\phi^{v}
$$

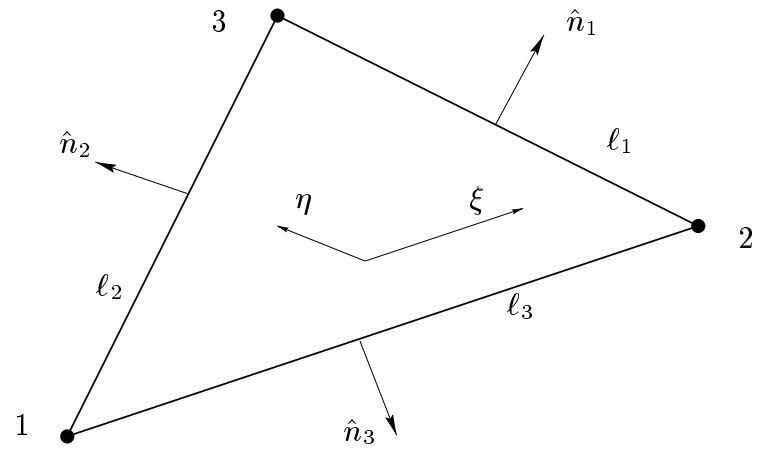

Fig. 1 Elemental triangular domain for fluctuation splitting.

The viscous fluctuation is assembled from contributions associated with each node of the triangle,

$$
\phi^{v}=\sum_{i=1}^{3} \phi_{i}^{v} \quad \text { and } \quad \phi_{i}^{v}=\frac{\ell_{i}}{2} \tilde{\vec{F}}^{v} \cdot \hat{n}_{i}
$$

The edge of the triangle opposite to node $i$ has length $\ell_{i}$ and outward unit normal $\hat{n}_{i}$.

Expressing the inviscid flux in terms of its Jacobian, $d \vec{F}=\vec{A} d U$, the inviscid fluctuation can be decomposed along two edges of a triangle, denoted $\xi$ for $\overline{12}$ and $\eta$ for $\overline{23}$, as

$$
\begin{gathered}
\phi=\phi^{\xi}+\phi^{\eta} \\
\phi^{\xi}=-\frac{1}{2} \ell_{1} \hat{n}_{1} \cdot \tilde{\vec{A} \widetilde{\Delta_{\xi}} U}, \quad \phi^{\eta}=\frac{1}{2} \ell_{3} \hat{n}_{3} \cdot \tilde{\vec{A}} \widetilde{\Delta_{\eta} U}
\end{gathered}
$$

A transformation to auxiliary variables, $d U=U_{W} d W$, simplifies the expression for the Jacobian,

$$
\begin{gathered}
\phi=\tilde{U}_{W} \breve{\phi} \\
\breve{\phi}=\breve{\phi}^{\xi}+\breve{\phi}^{\eta}=-\alpha \widetilde{\Delta_{\xi} W}-\beta \widetilde{\Delta_{\eta} W}
\end{gathered}
$$

with

$$
\begin{gathered}
U_{W}=\left[\begin{array}{cccc}
1 & 0 & 0 & \frac{1}{a^{2}} \\
u & 1 & 0 & \frac{1}{a^{2}} u \\
v & 0 & 1 & \frac{1}{a^{2}} v \\
\frac{V^{2}}{2} & u & v & \frac{1}{\gamma-1} \frac{T_{0}}{T}
\end{array}\right] \\
\alpha=\frac{1}{2} \ell_{1} \hat{n}_{1} \cdot \tilde{\overrightarrow{\mathcal{A}}}, \quad \beta=-\frac{1}{2} \ell_{3} \hat{n}_{3} \cdot \tilde{\overrightarrow{\mathcal{A}}}
\end{gathered}
$$

and

$$
\overrightarrow{\mathcal{A}}=\vec{V} I+\left[\begin{array}{cccc}
0 & 0 & 0 & 0 \\
0 & 0 & 0 & \hat{\imath} \\
0 & 0 & 0 & \hat{\jmath} \\
0 & a^{2} \hat{\imath} & a^{2} \hat{\jmath} & 0
\end{array}\right],
$$

Upwinding is achieved through the introduction of an artificial dissipation fluctuation,

$$
\breve{\phi}^{\prime \xi}=\operatorname{sign}\left(\hat{n}_{1} \cdot \tilde{\overrightarrow{\mathcal{A}}}\right) \breve{\phi}^{\xi}, \quad \breve{\phi}^{\prime \eta}=\operatorname{sign}\left(-\hat{n}_{3} \cdot \tilde{\overrightarrow{\mathcal{A}}}\right) \breve{\phi}^{\eta}
$$

which is linearly combined with the basic fluctuation to complete the inviscid contribution to the nodal updates. 


\section{Adaption Strategy}

The present adaption strategy for two-dimensional unstructured meshes with triangular elements is local in scope and allows anisotropic stretching. Adaption cycles are performed as sequential sweeps over the domain performing the basic operations: node deletion, edge swapping, nodal displacement, and node insertion. The process is automated and a variety of stopping criteria can be considered, such as a maximum grid size, total number of cycles, or minimum to maximum residual ratio.

A node is marked for deletion if the fluctuations, both inviscid and viscous, ${ }^{a}$ in all surrounding cells are below a threshold. The threshold is customizable, and a typical choice would be $2-3$ orders of magnitude below the average nodal value. A trial reconnection of the mesh is proposed without the node and the element fluctuations are recomputed, from the existing solution, to verify that the fluctuations remain small. If so, the node deletion is finalized.

A node is added at the midpoint of an edge, creating two more cells and three more edges, if the fluctuations in the cells to either side of the edge exceed a threshold. When comparing cell fluctuations, the $\mathrm{L}_{2}$-norm of the fluctuations from each of the governing equations are used, weighted by the inverse of the square root of the cell area.

Edges are flagged to be swapped when the root mean square (RMS) of the fluctuations in the cells to either side are excessive. If the swapped edge maintains a valid grid then the RMS of the fluctuations is recomputed, and the swapped configuration is retained if the RMS has decreased.

Nodal displacements are driven by the minimization of a functional that can be expressed in terms of either the conserved or auxiliary fluctuations, as the minimization of one implies the minimization of the other. Because the goal of the nodal displacements is to achieve alignment of the mesh with inviscid discontinuities and characteristic lines, the viscous fluctuation is omitted from the displacement functional.

The nodal displacement functional is constructed for each node $i$ as

$$
\Upsilon_{i}=\frac{1}{2} \sum_{\mathrm{T}} \breve{\phi}_{\mathrm{T}}^{T} \Xi_{\mathrm{T}} \breve{\phi}_{\mathrm{T}}
$$

where the summation is over all triangles connected at node $i$ and $\Xi$ is the weighting matrix. The derivatives

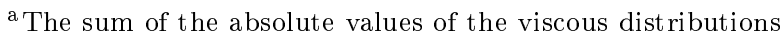
is used, as the net cellular viscous fluctuation is identically zero. of $\Upsilon$ are formed using the chain rule as

$$
\begin{array}{r}
\frac{\partial \Upsilon_{i}}{\partial x_{i}}=\frac{1}{2} \sum_{\mathrm{T}}\left[\left(\frac{\partial \breve{\phi}_{\mathrm{T}}}{\partial x_{i}}\right)^{T} \Xi_{\mathrm{T}} \breve{\phi}_{\mathrm{T}}+\breve{\phi}_{\mathrm{T}} \frac{\partial \Xi_{\mathrm{T}}}{\partial x_{i}} \breve{\phi}_{\mathrm{T}}\right. \\
\left.+\breve{\phi}_{\mathrm{T}}^{T} \Xi_{\mathrm{T}} \frac{\partial \breve{\phi}_{\mathrm{T}}}{\partial x_{i}}\right],
\end{array}
$$

and

$$
\begin{array}{r}
\frac{\partial \Upsilon_{i}}{\partial y_{i}}=\frac{1}{2} \sum_{\mathrm{T}}\left[\left(\frac{\partial \breve{\phi}_{\mathrm{T}}}{\partial y_{i}}\right)^{T} \Xi_{\mathrm{T}} \breve{\phi}_{\mathrm{T}}+\breve{\phi}_{\mathrm{T}} \frac{\partial \Xi_{\mathrm{T}}}{\partial y_{i}} \breve{\phi}_{\mathrm{T}}\right. \\
\left.+\breve{\phi}_{\mathrm{T}}^{T} \Xi_{\mathrm{T}} \frac{\partial \breve{\phi}_{\mathrm{T}}}{\partial y_{i}}\right] .
\end{array}
$$

Having defined the gradient of the functional, the method of steepest descent can be applied directly to drive the nodal displacements.

The weighting factor $\Xi_{\mathrm{T}}$ is a symmetric positivedefinite matrix. Weighting each equation equally without regard to cell sizes results in $\Xi_{\mathrm{T}}=I$, while an inverse area weighting yields $\Xi_{\mathrm{T}}=\frac{1}{S_{\mathrm{T}}} I$. The derivatives of $\Xi_{\mathrm{T}}$ for these and other area-weighted choices are available in Ref. 14. The present study is conducted using equal weighting.

The discrete cell fluctuation, Eqn. 7, can be equivalently written as

$$
\breve{\phi}=\frac{1}{2} \sum_{j=1}^{3} \ell_{j} \hat{n}_{j} \cdot \tilde{\overrightarrow{\mathcal{A}}} W_{j},
$$

where $W_{j}=W_{\bar{Z}} Z_{j}$. The summation is over the three nodes defining the triangle.

The derivatives of the fluctuation with respect to the movement of only one node, for example node 2 of the triangle, are

$$
\begin{aligned}
\frac{\partial \breve{\phi}}{\partial x_{2}}= & \frac{1}{2}\left[\tilde{\mathcal{A}}^{\mathrm{y}}\left(W_{1}-W_{3}\right)\right. \\
& \left.+\sum_{j=1}^{3} \ell_{j} \hat{n}_{j}\left(\frac{\partial \tilde{\overrightarrow{\mathcal{A}}}}{\partial x_{2}} W_{j}+\tilde{\overrightarrow{\mathcal{A}}} \frac{\partial W_{j}}{\partial x_{2}}\right)\right],
\end{aligned}
$$

and

$$
\begin{aligned}
\frac{\partial \breve{\phi}}{\partial y_{2}}= & \frac{1}{2}\left[\tilde{\mathcal{A}}^{\mathrm{x}}\left(W_{3}-W_{1}\right)\right. \\
& \left.+\sum_{j=1}^{3} \ell_{j} \hat{n}_{j}\left(\frac{\partial \tilde{\overrightarrow{\mathcal{A}}}}{\partial y_{2}} W_{j}+\tilde{\overrightarrow{\mathcal{A}}} \frac{\partial W_{j}}{\partial y_{2}}\right)\right] .
\end{aligned}
$$

The derivatives of the fluctuation with respect to movements of node 1 or 3 of the triangle follow directly as cyclic permutations. 
The flux Jacobian in auxiliary variables, Eqn. 10, has the approximate derivative

$$
\frac{\partial \overrightarrow{\mathcal{A}}}{\partial x} \simeq \frac{\partial \vec{V}}{\partial x} I
$$

and similarly for the $y$ derivatives. It is assumed that moving a node does not change the solution at the other two nodes of the triangle, so that the variation of the cell-average flux Jacobian scales like one-third the variation of the velocity at the node being moved,

$$
\frac{\partial \tilde{\overrightarrow{\mathcal{A}}}}{\partial x_{2}} \simeq \frac{1}{3} \frac{\partial \vec{V}_{2}}{\partial x_{2}} I
$$

Similarly, the variation of the Jacobian of the transformation scales like one-third of the nodal variation, $\frac{\partial W_{\bar{Z}}}{\partial x_{2}} \sim \frac{1}{3} \frac{\partial Z_{2}}{\partial x_{2}}$, and is neglected as a sub-principle term relative to the change in solution value,

$$
\frac{\partial W_{j}}{\partial x_{2}} \simeq W_{\bar{Z}} \frac{\partial Z_{j}}{\partial x_{2}} .
$$

Since the solution is locally assumed to vary only at the node being moved, i.e., $\frac{\partial Z_{1}}{\partial x_{2}}=\frac{\partial Z_{3}}{\partial x_{2}}=0$,

$$
\sum_{j=1}^{3} \frac{\partial W_{j}}{\partial x_{2}} \simeq W_{\bar{Z}} \frac{\partial Z_{2}}{\partial x_{2}} .
$$

The remaining term to evaluate is $\frac{\partial Z_{2}}{\partial x_{2}}$. The steadystate distribution of the residual fluctuation to the node can be written

$$
\begin{aligned}
\sum_{\mathbf{T}}\left[\left(I+\mathbf{M}_{\alpha}\right) \alpha-\left(I+\mathbf{M}_{\beta}\right) \beta\right] W_{\bar{Z}} Z_{2} \\
=\sum_{\mathbf{T}}\left[\left(I+\mathbf{M}_{\alpha}\right) \alpha W_{\bar{Z}} Z_{1}-\left(I+\mathbf{M}_{\beta}\right) \beta W_{\bar{Z}} Z_{3}\right],
\end{aligned}
$$

or

$$
\sum_{\mathrm{T}}\left(\alpha^{+}-\beta^{+}\right) W_{\bar{Z}} Z_{2}=\sum_{\mathrm{T}}\left(\alpha^{+} W_{\bar{Z}} Z_{1}-\beta^{+} W_{\bar{Z}} Z_{3}\right),
$$

where

$$
\begin{aligned}
\alpha^{+} & =\frac{1}{2}\left(I+\mathbf{M}_{\alpha}\right) \alpha, \\
\beta^{+} & =\frac{1}{2}\left(I+\mathbf{M}_{\beta}\right) \beta .
\end{aligned}
$$

The upwinding matrices $\mathbf{M}_{\alpha}$ and $\mathbf{M}_{\beta}$ expressed in auxiliary variables reduce to the identity matrix for supersonic flow, see Ref. 13. Differentiating Eqn. 23 while freezing the Jacobians leads to

$$
\begin{aligned}
\sum_{\mathrm{T}} & {\left[\left(\frac{\partial \alpha^{+}}{\partial x_{2}}-\frac{\partial \beta^{+}}{\partial x_{2}}\right) W_{\bar{Z}} Z_{2}+\left(\alpha^{+}-\beta^{+}\right) W_{\bar{Z}} \frac{\partial Z_{2}}{\partial x_{2}}\right] } \\
& =\sum_{\mathrm{T}}\left(\frac{\partial \alpha^{+}}{\partial x_{2}} W_{\bar{Z}} Z_{1}-\frac{\partial \beta^{+}}{\partial x_{2}} W_{\bar{Z}} Z_{3}\right),
\end{aligned}
$$

$$
\begin{aligned}
& \sum_{\mathrm{T}}\left(\alpha^{+}-\beta^{+}\right) W_{\bar{Z}} \frac{\partial Z_{2}}{\partial x_{2}} \\
&=\sum_{\mathrm{T}}\left[\frac{\partial \alpha^{+}}{\partial x_{2}} W_{\bar{Z}}\left(Z_{1}-Z_{2}\right)\right. \\
&\left.\quad-\frac{\partial \beta^{+}}{\partial x_{2}} W_{\bar{Z}}\left(Z_{3}-Z_{2}\right)\right],
\end{aligned}
$$

and

$$
\begin{aligned}
\frac{\partial Z_{2}}{\partial x_{2}}=- & {\left[\sum_{\mathrm{T}}\left(\alpha^{+}-\beta^{+}\right) W_{\bar{Z}}\right]^{-1} } \\
& \sum_{\mathrm{T}}\left(\frac{\partial \alpha^{+}}{\partial x_{2}} \widetilde{\Delta_{\xi} W}+\frac{\partial \beta^{+}}{\partial x_{2}} \widetilde{\Delta_{\eta} W}\right) .
\end{aligned}
$$

A similar expression can be formed for the $y$ derivative.

Neglecting the subsonic blending on $\mathbf{M}_{\alpha}$ and $\mathbf{M}_{\beta}$ allows $\alpha^{+}$and $\beta^{+}$to be expressed as

$$
\alpha^{+}=\left\{\begin{array}{cc}
\alpha, & \tilde{\mathcal{V}}_{\alpha}>0 \\
0, & \tilde{\mathcal{V}}_{\alpha} \leq 0
\end{array}, \quad \beta^{+}=\left\{\begin{array}{cc}
\beta, & \tilde{\mathcal{V}}_{\beta}>0 \\
0, & \tilde{\mathcal{V}}_{\beta} \leq 0
\end{array},\right.\right.
$$

where $\tilde{\mathcal{V}}_{\alpha}=\vec{V} \cdot \hat{n}_{1}$ and $\tilde{\mathcal{V}}_{\beta}=\vec{V} \cdot \hat{n}_{3}$. The derivatives follow directly,

$$
\begin{aligned}
& \frac{\partial \alpha^{+}}{\partial x_{2}}=\left\{\begin{array}{cc}
\frac{\partial \alpha}{\partial x_{2}}, & \tilde{\mathcal{V}}_{\alpha}>0 \\
0, & \tilde{\mathcal{V}}_{\alpha} \leq 0
\end{array},\right. \\
& \frac{\partial \beta^{+}}{\partial x_{2}}=\left\{\begin{array}{cc}
\frac{\partial \beta}{\partial x_{2}}, & \tilde{\mathcal{V}}_{\beta}>0 \\
0, & \tilde{\mathcal{V}}_{\beta} \leq 0
\end{array} .\right.
\end{aligned}
$$

The derivatives of $\alpha$ and $\beta$ are

$$
\begin{aligned}
\frac{\partial \alpha}{\partial x_{2}} & =\frac{\tilde{\mathcal{A}}^{\mathrm{y}}}{2}+\frac{1}{2} \ell_{1} \hat{n}_{1} \cdot \frac{\partial \tilde{\overrightarrow{\mathcal{A}}}}{\partial x_{2}}, \\
\frac{\partial \alpha}{\partial y_{2}} & =-\frac{\tilde{\mathcal{A}}^{\mathrm{x}}}{2}+\frac{1}{2} \ell_{1} \hat{n}_{1} \cdot \frac{\partial \overrightarrow{\overrightarrow{\mathcal{A}}}}{\partial y_{2}}, \\
\frac{\partial \beta}{\partial x_{2}} & =\frac{\tilde{\mathcal{A}}^{\mathrm{y}}}{2}-\frac{1}{2} \ell_{3} \hat{n}_{3} \cdot \frac{\partial \tilde{\overrightarrow{\mathcal{A}}}}{\partial x_{2}}, \\
\frac{\partial \beta}{\partial y_{2}} & =-\frac{\tilde{\mathcal{A}}^{\mathrm{x}}}{2}-\frac{1}{2} \ell_{3} \hat{n}_{3} \cdot \frac{\partial \overrightarrow{\overrightarrow{\mathcal{A}}}}{\partial y_{2}} .
\end{aligned}
$$

Having determined $\frac{\partial Z_{2}}{\partial x_{2}}, \frac{\partial \vec{V}_{2}}{\partial x_{2}}$ follows from

$$
d u=\frac{d(\sqrt{\rho} u)-u d(\sqrt{\rho})}{\sqrt{\rho}},
$$

and

$$
d v=\frac{d(\sqrt{\rho} v)-v d(\sqrt{\rho})}{\sqrt{\rho}} .
$$

This completes the derivation of all the terms required to evaluate the derivatives of the two-dimensional objective function, Eqns. 13 and 14. Reference 6 presents the analogous derivation in axisymmetric coordinates. 


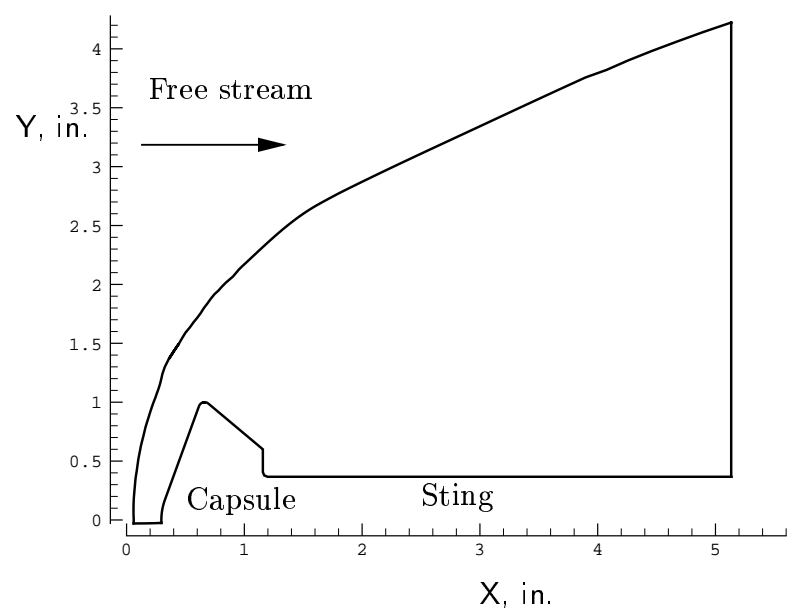

Fig. 2 Computational domain for Mars Pathfinder capsule.

\section{Case Definition}

Evaluation of the adaption strategy for hypersonic flow is simplified by choosing a two-dimensional laminar perfect gas case. The test conditions and geometry correspond to the Mach-10 wind tunnel tests of Hollis. ${ }^{15}$ The sting-mounted capsule geometry, depicted in Figure 2, is modeled as two-dimensional instead of axisymmetric for the present study. ${ }^{\mathrm{b}}$ The original axisymmetric wind tunnel model of the Mars Pathfinder capsule consists of a spherically-blunted 70degree sphere-cone. The body radius is 1 in. and the nose radius is $\frac{1}{2}$ in. The shoulder radius is $\frac{1}{20} \mathrm{in}$. and the aft body angle is 40 degrees. The base radius is $\frac{3}{5}$ in. The sting is $4 \mathrm{in}$. long with a radius of $\frac{13}{32} \mathrm{in}$.

The free stream conditions for the NASA Langley 31-Inch Mach 10 Air Tunnel, corresponding to a nominal Reynolds number per foot of $0.5 \times 10^{6}$, are: $P=69 \mathrm{~Pa}, T=53 \mathrm{~K}, \rho=0.0045 \mathrm{~kg} / \mathrm{m}^{3}$, and $u=1416 \mathrm{~m} / \mathrm{s}$. The wall temperature is taken to be a uniform $300 \mathrm{~K}$. Historical experience with this tunnel at these conditions indicates that laminar perfect gas calculations are adequate for comparison with the experimental data.

The metric for accuracy is chosen to be surface heat transfer rates. The benchmark solution is obtained using the cell-centered flux difference splitting secondorder accurate LAURA code of Gnoffo. ${ }^{16}$ A structured grid with 125 stream-wise nodes and 513 nodes from the surface to the outer boundary, for a total of 64,125 nodes, is used to generate the benchmark solution. The grid is adapted for alignment with the bow shock and clustered to the boundary layer. The benchmark solution is shown to be grid converged on this mesh, aside from small changes at the stagnation point, in

\footnotetext{
${ }^{\mathrm{b}}$ Reference 6 reports the axisymmetric results for this case, with similar observed behavior and conclusions as for the twodimensional results, but with the added complexity of special treatment for the axis singularity.
}

Ref. 6. The same reference also shows the grid convergence of the present solver for this case using uniform mesh refinement, converging in agreement with the benchmark solution.

\section{Adaption Component Evaluation}

The individual adaption operations, deletion, swapping, moving, and insertion, are first tested separately to gage their relative merits. Each evaluation begins from a converged solution on triangulations of coarsened versions of the structured LAURA grid. After adaption the solution is re-converged. The adaptation will be considered successful if the resultant surface heat transfer rates are closer to the benchmark data.

For point deletion the starting mesh is a triangulation of $125 \times 257$ structured grid, containing 32,125 nodes. The objective for successful point deletion is to remove points without altering the solution. For the first pass, the adaption is set to remove 10 percent (3284) of the nodes, producing minimal change to the solution. The nodes are predominantly removed from the free stream. Then a further 10 percent (3157) of the nodes are removed, and the heating rates remain essentially unchanged, Figure 3, but the fluctuation splitting scheme has trouble maintaining the bow shock capture due to the further loss of free stream points, as shown in Figure 4. The removal of more points causes rapid solution deterioration as the scheme fails to properly capture the bow shock on the coarsened meshes.

For evaluating edge swapping the starting mesh is formed from a triangulated $125 \times 129$ structured grid with 16,125 nodes and 47,868 edges. Edge swapping with the fluctuation minimization strategy is not beneficial for this case. Only about 1 percent of the edges are swapped, primarily at the shock, but the resulting solution does not converge due to ringing of the bow shock near the stagnation point. Figure 5 shows pressure contours in the stagnation point region overlaid upon the swapped mesh. Notice the irregular contours downstream of the shock. The difficulty in this solution is that the bow shock is making discrete jumps across grid lines, induced by swapped edges at the shock. The unadapted bow shock more closely follows the grid lines at the $y=0$ symmetry plane. Recall that this mesh was originally well-aligned with the bow shock from the structured-grid solver, and the effect of edge swapping on a more random unstructured mesh may be different. Also, on a finer mesh the smaller grid spacing at the shock might lessen the detrimental effects of discrete jumps in the shock location. The edge swapping assessment is tried again with more conservative thresholds, this time only swapping half a percent of the edges, but still produces the same disappointing results.

Nodal displacements are evaluated using the 16,125node mesh. The adaption moves 1827 nodes a total 


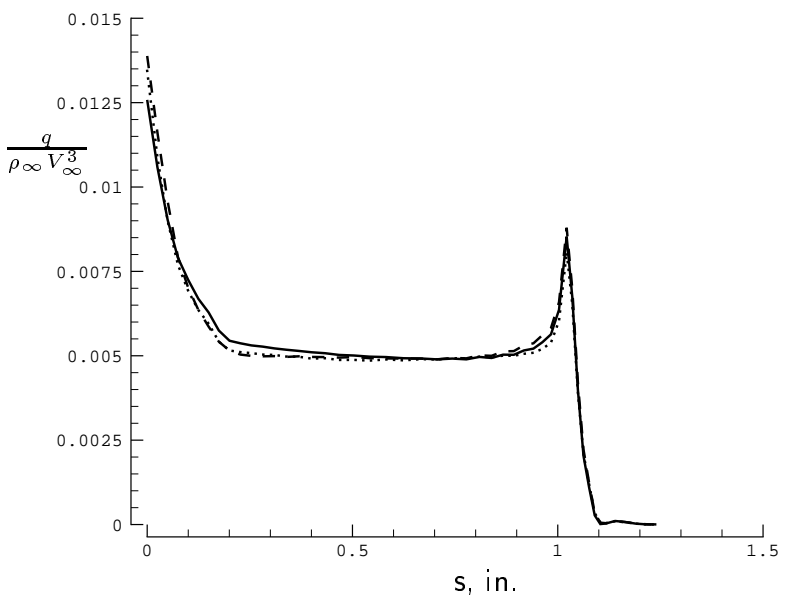

a) Heat shield.

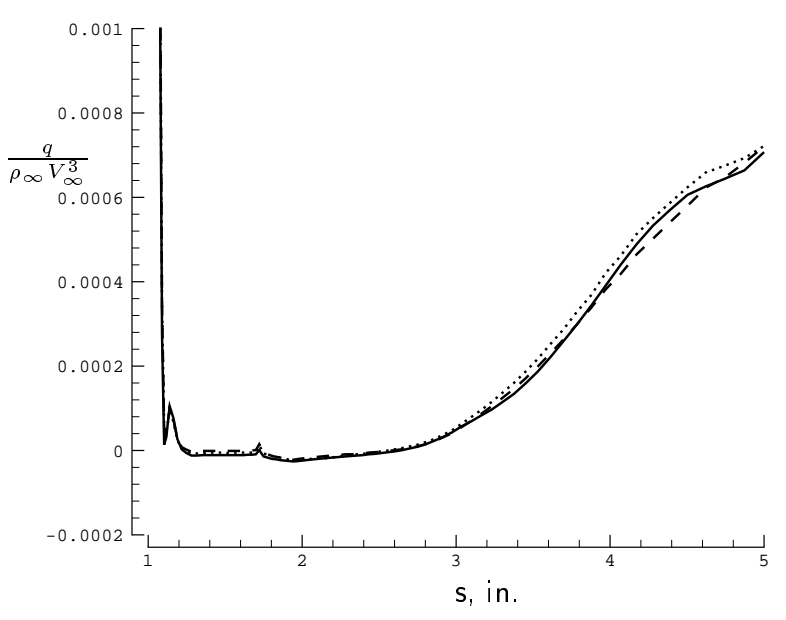

b) Aft body and sting.

Fig. 3 Surface heating after coarsening, solid $=$ unadapted, dashed $=10 \%$ removed, dotted $=20 \%$ removed.

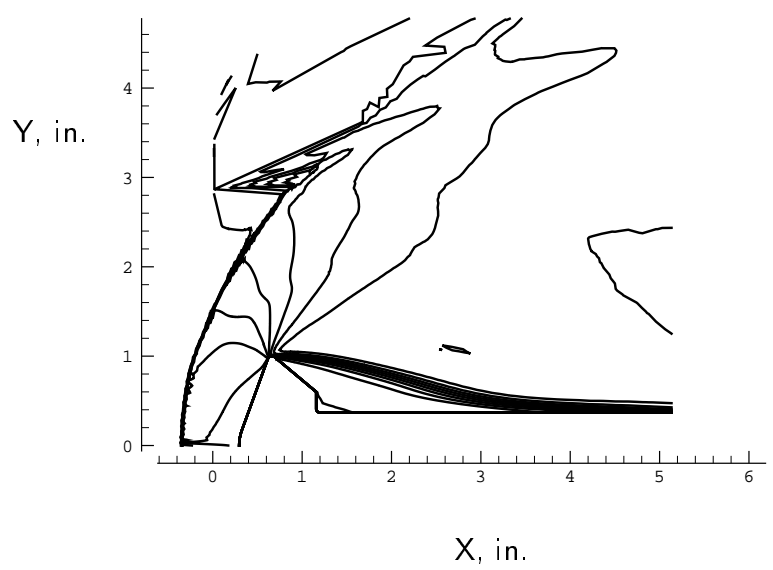

Fig. 4 Loss of shock capture due to coarsening, pressure contours.

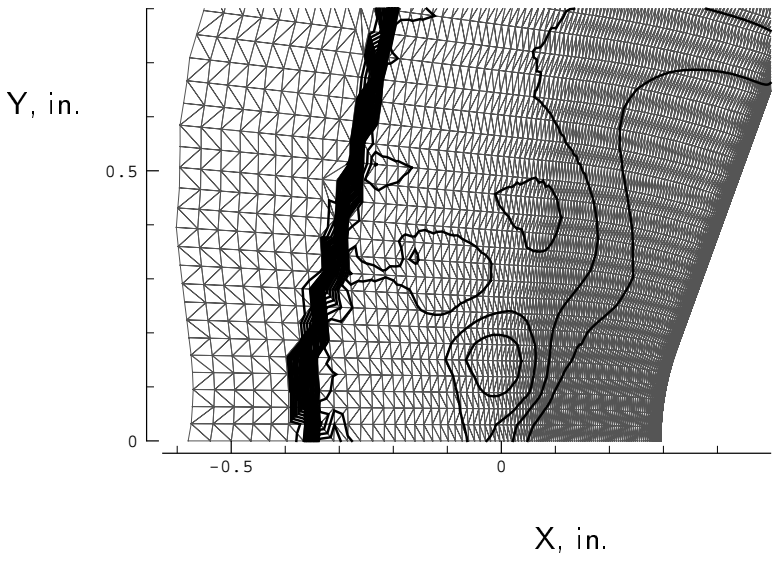

Fig. 5 Shock bulging at stagnation point after edge swaps, pressure contours and grid.

distance of 0.7488 in., for an average of 0.0004 in. and RMS of 0.0015 in. This level of movement represents a perturbation to the mesh, with the average movement being an order of magnitude smaller than the edge lengths at the shock. The mesh movement occurs at the shock, near the shoulder, and in the fore body boundary layer. Surface heating rates for this case are shown in Figure 6. The solution is worsened on the fore body while not much change is seen in the wake. Investigating the solution reveals that a wind side vortex pattern has emerged, Figure 7, that is causing the unexpected heat transfer rates on the heat shield. The solution is run a further 800,000 iterations with no change to this wind side vortex pattern. Note that the solver includes eigenvalue limiting, which is known to suppress the carbuncle phenomenon for the structured grid Roe schemes. The wind side recirculation is not a physically accurate prediction nor does it appear to match the classic carbuncle pathology, but is rather an artifact of discrete mesh point jumps in the bow shock location, seen in Figure 7 as choppiness in the bow shock contour lines. For this case the adaption perturbs a mesh that was initially well aligned to the bow shock in such a way as to largely destroy that alignment, to the detriment of the solution.

Point insertion is tested on the unadapted 16,125node mesh. The adaptation adds 3299 nodes, approximately a 20 percent increase, with heating results shown in Figure 8. Many of these new points are added to the wind side boundary layer. On the heat shield the heating levels rise markedly toward the benchmark result, but exhibit a high-frequency oscillation of significant amplitude. There is little change in the wake heating.

\section{Full Adaption Cycle Evaluation}

For the full adaption the starting solution is taken on the triangulated $125 \times 129$ mesh with 16,125 nodes. The intention is to look for an improvement in the 


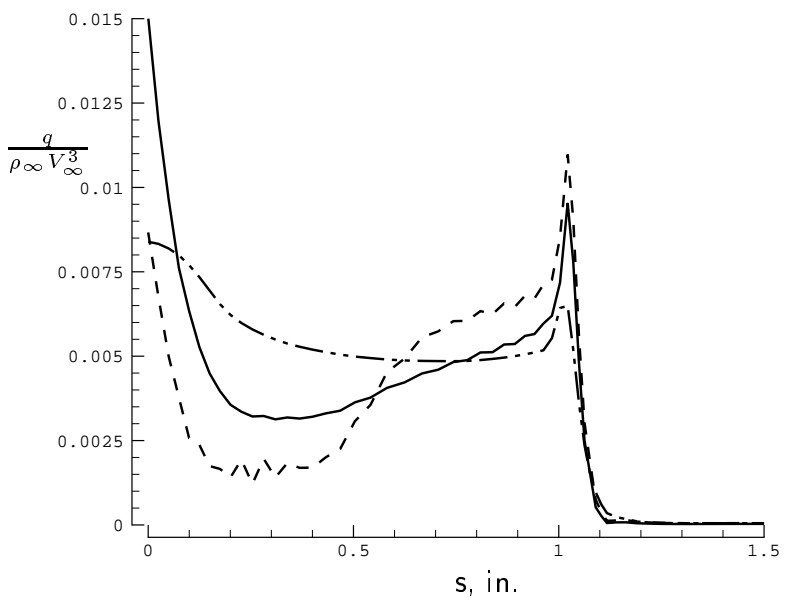

a) Heat shield.

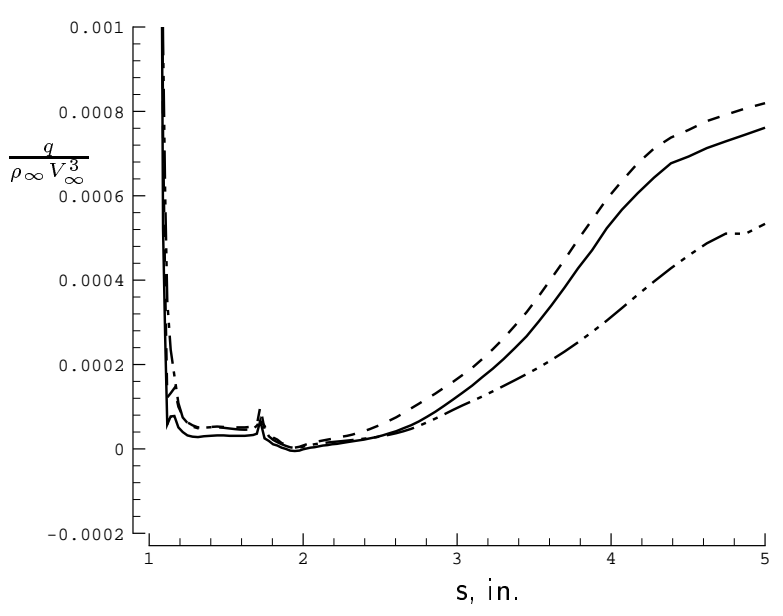

b) Aft body and sting.

Fig. 6 Surface heating after nodal displacements, solid=unadapted, dashed $=10 \%$ moved, dash-dotdot=benchmark.

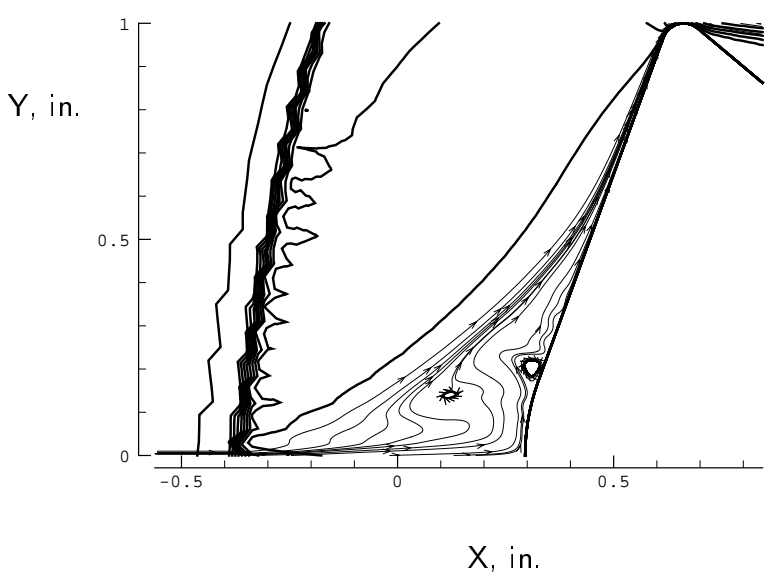

Fig. 7 Wind Side vortices produced by nodal displacements, streamlines and pressure contours.

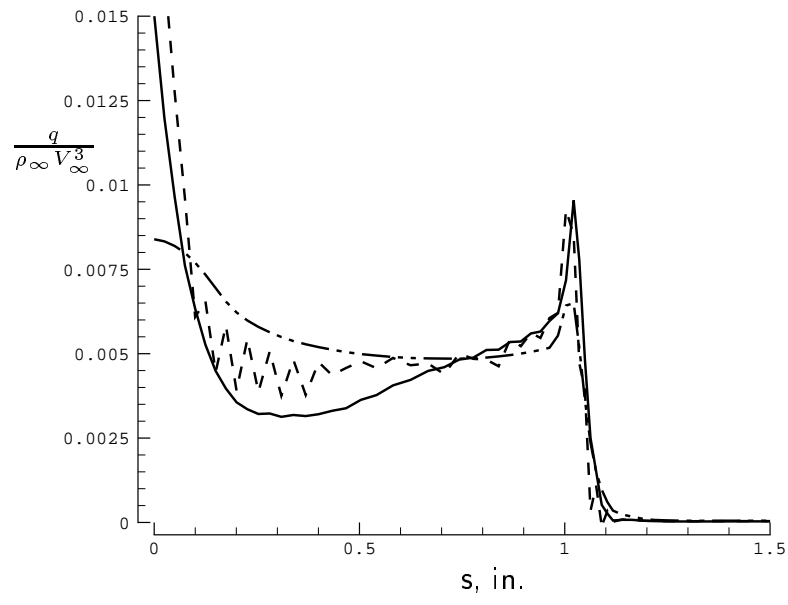

a) Heat shield.

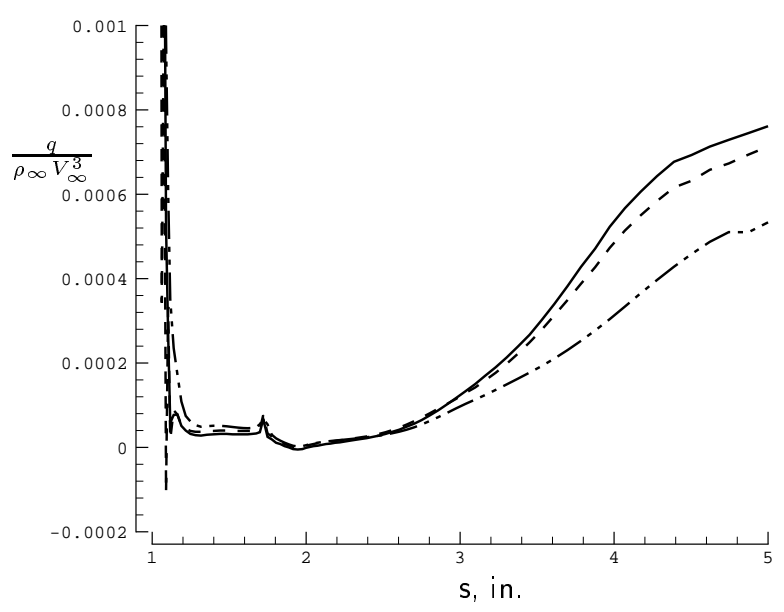

b) Aft body and sting.

Fig. 8 Surface heating after point insertion, solid $=$ unadapted, dashed $=20 \%$ inserted, dash-dotdot=benchmark.

coarse mesh solution without increasing the number of nodes. Although not all of the component adaptation steps were seen to be beneficial, a complete cycle will include each step to allow for synergism between the components. The strategy for an adaption cycle is to delete 10 percent of the nodes, swap 1 percent of the edges, move 5 percent of the nodes, and then insert back in 10 percent of the nodes. The solution is reconverged between each step of the adaption cycle.

The fluctuation minimization adaption successfully removes 1601 nodes, but runs into trouble again while swapping. Wind side vortices are spawned in the stagnation region by an oscillating bow shock. In an effort to damp the solution the CFL number is reduced by an order of magnitude, without producing an improvement in the solution or eliminating the vortices. The number of edges to be swapped is then reduced to $\frac{1}{2}$ percent, but the same vortices and oscillating bow 


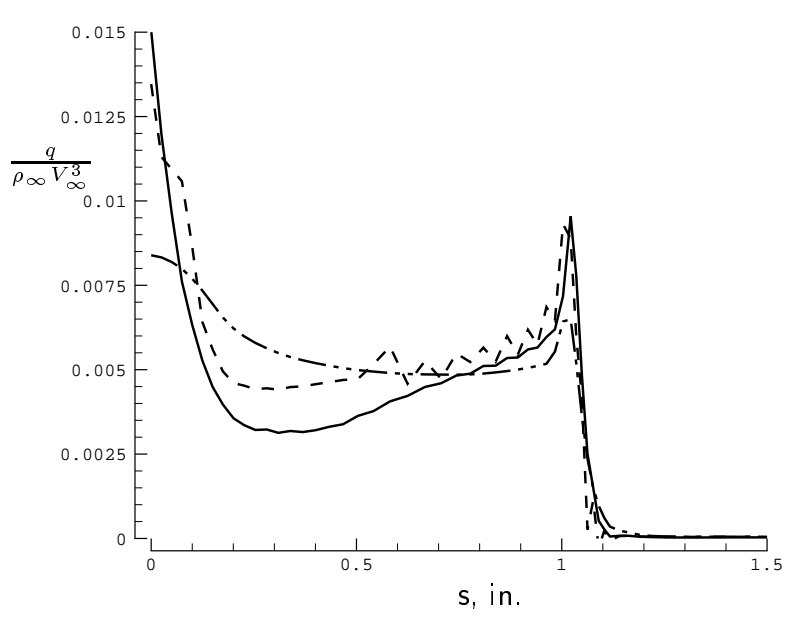

a) Heat shield.

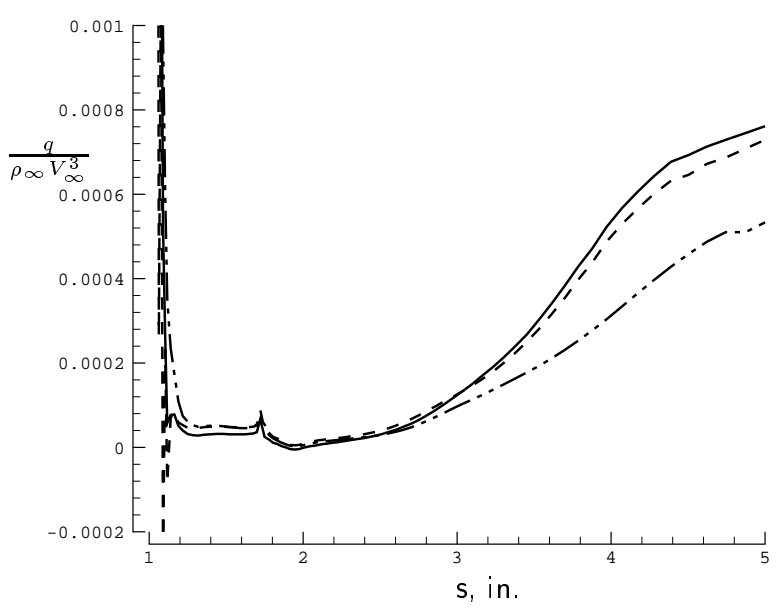

b) Aft body and sting.

Fig. 9 Surface heating after full adaption cycle, solid=unadapted, dashed=adapted, dash-dotdot=benchmark.

shock appear. To proceed with this case the edge swapping is omitted entirely and the adaption cycle continued with the nodal displacement step, where 706 nodes are moved a total distance of 0.7 in. Finally, 1638 nodes are added, yielding the results of Figure 9. The fore body heating is generally improved toward the benchmark solution, although there is a high-frequency oscillation in the data starting at $s=0.6$. The aft body heating is improved to match the benchmark between $s=1.2-1.8$, although the original solution was not far off from the benchmark in this region. The sting heating is only slightly changed aft of $s=3$.

\section{Concluding Remarks}

An anisotropic unstructured mesh adaption strategy for hypersonic viscous fluid flows is presented. The strategy is implemented for two-dimensional perfect gas laminar physics as a prototype for the purpose of assessing the benefits of the scheme prior to pursuing in a three-dimensional reacting gas implementation. The adaption criteria is based upon the minimization of residual fluctuations defined by a multidimensional upwind distribution scheme for the flow solver. Particular emphasis is placed on deriving the derivatives of the nodal displacement objective function for the upwind fluctuation splitting distribution algorithm.

The hypersonic demonstration case is a two-dimensional sting-mounted capsule at laminar Mach 10 wind tunnel conditions, modeled with perfect gas air. Surface heat transfer is the quantity of interest used to measure the success of the solution adaptive process.

Four elementary operations are performed during the adaption: point deletion, edge swapping, nodal displacement, and point insertion. The limits of effectiveness for each of the four operations is probed independently to guide their relative weighting in the full adaption strategy. Coarsening of the mesh works well removing up to about 20 percent of the nodes, but leads to a loss of the bow shock capture and a rapidly deteriorating solution for more aggressive coarsening. The other three adaption techniques generally have minimal or negative impacts on the solution, with the exception of point insertion where solution improvement occurs but at the cost of high frequency oscillations. Overall a lack of robustness is demonstrated, usually caused by distortions to the bow shock near the stagnation point. Common failure modes are an oscillating shock that sheds wind side vortices, or a steady shock kink that produces unexpected pressure contours and surface heating spikes. For the full adaption cycle the fore body heating improves but becomes oscillatory, while the aft body and sting heating predictions improve slightly.

One likely cause of the lack of robustness exhibited by the adaption scheme at the stagnation point bow shock is the conflict between characteristic directions between the supersonic upstream flow, which is perfectly upwinded by the flow solver, and the subsonic shock layer, where the acoustic characteristics approximated as discrete waves. A second contributor to the lack of robustness is the local scope of the adaption strategy. The scheme is intentionally local to be synergistic with the flow solver which has compact support, sharing the same software data structures and loop controls. The local scope also is desirable for parallel processing. However, a larger stencil, an implicit scheme, or an adjoint equation criteria may be necessary to provide sufficient smoothness at shocks.

Although heat transfer is a variable of primary interest for aerothermodynamics and may be a first consideration for driving the adaption criteria, the present results emphasize that for blunt-body hypersonic aerothermodynamics both the bow shock location and smoothness are critical to the computation of 
the fore body heat transfer rates. Due to the strength of the hypersonic bow shock, discrete jumps in shock position from one node to another produce significant pressure disturbance waves and entropy variations that strongly affect the flow field in the subsonic bubble and overwhelm more subtle adaption within the boundary layer.

To summarize, the present adaption techniques do not significantly improve the solutions, the adaption effectiveness is not consistent, and the solutions display a lack of robustness. Point deletion works the best, but is risky if applied too aggressively. Uniform refinement is both simpler and more reliable than the solution adaptive strategy embodied by the present method.

\section{References}

${ }^{1}$ Thomas, J. L., Alexandrov, N., Alter, S. J., Atkins, H. L., Bey, K. S., Bibb, K. L., Biedron, R. T., Carpenter, M. H., Cheatwood, F. M., Drummond, P. J., Gnoffo, P. A., Jones, W. T., Kleb, W. L., Lee-Rausch, E. M., Merski, N. R., Mineck, R. E., Nielsen, E. J., Park, M. A., Pirzadeh, S. Z., Roberts, T. W., Samareh, J. A., Swanson, R. C., Vatsa, V. N., Weilmuenster, K. J., White, J. A., Wood, W. A., and Yip, L. P., "Opportunities for Breakthroughs in Large-Scale Computational Simulation and Design," NASA/TM 2002-211747, June 2002.

${ }^{2}$ Venditti, D. A. and Darmofal, D. L., "Grid Adaptation for Functional Outputs: Application to Two-Dimensional Inviscid Flows," Journal of Computational Physics, Vol. 176, No. 1, Feb. 2002, pp. 40-69.

${ }^{3}$ Habashi, W. G., Fortin, M., Dompierre, J., Vallet, M.-G., and Bourgault, Y., "Anisotropic Mesh Adaption: A Step Towards a Mesh-Independent and User-Independent CFD," Barriers and Challenges in Computational Fluid Dynamics, edited by V. Venkatakrishnan et al., Kluwer Academic Publisher, 1998, pp. 99-117.

${ }^{4}$ Habashi, W. G., Dompierre, J., Bourgault, Y., Fortin, M., and Vallet, M.-G., "Certifiable Computational Fluid Dynamics Through Mesh Optimization," AIA A Journal, Vol. 36, No. 5, May 1998, pp. 703-711.

${ }^{5}$ Wood, W. A. and Kleb, W. L., "Diffusion Characteristics of Finite Volume and Fluctuation Splitting Schemes," Journal of Computational Physics, Vol. 153, No. 2, Aug. 1999, pp. 353377.

${ }^{6}$ Wood, W. A., "Multi-dimensional Upwind Fluctuation Splitting Scheme with Mesh Adaption for Hypersonic Viscous Flow," NASA/TP 2002-211640, April 2002.

${ }^{7}$ Caraeni, D., Caraeni, M., and Fuchs, L., "A Parallel Multidimensional Upwind Algorithm for LES," AIAA 2001-2547, June 2001.

${ }^{8}$ Roe, P., "Compounded of Many Simples," Barriers and Challenges in Computational Fluid Dynamics, edited by V. Venkatakrishnan et al., Kluwer Academic Publishers, 1998, pp. 241-258.

${ }^{9}$ Roe, P. and Nishikawa, H., "Adaptive Grid Generation by Minimising Residuals," ICFD Conference on Numerical Methods in Fluid Dynamics, Oxford, U.K., 2001, submitted to the International Journal for Numerical Methods in Fluids.

${ }^{10}$ Wood, W. A. and Kleb, W. L., "On Multi-dimensional Ustructured Mesh Adaption," AIAA Paper 99-3254, June 1999.

${ }^{11}$ Yamaleev, N. K. and Carpenter, M. H., "On Accuracy of Adaptive Grid Methods for Captured Shocks," submitted to Journal of Computational Physics, 2001.

${ }^{12}$ Sidilkover, D., "Multidimensional Upwinding and Multigrid," AIAA Paper 95-1759, June 1995.
${ }^{13}$ Wood, W. A. and Kleb, W. L., "2-D/Axisymmetric Formulation of Multi-dimensional Upwind Scheme," AIAA Paper 2001-2630, June 2001.

${ }^{14}$ Wood, W. A., Multi-dimensional Upwind Fluctuation Splitting Scheme with Mesh Adaption for Hypersonic Viscous Flow, Ph.D. thesis, Virginia Tech, 2001.

${ }^{15}$ Hollis, B. R. and Perkins, J. N., "Transition Effects on Heating in the Wake of a Blunt Body," Journal of Spacecraft and Rockets, Vol. 36, No. 5, Sept. 1999, pp. 668-674.

${ }^{16}$ Gnoffo, P. A., Gupta, R. N., and Shinn, J. L., "Conservation Equations and Physical Models for Hypersonic Air Flows in Thermal and Chemical Nonequilibrium," NASA TP 2867, Feb. 1989. 